\title{
Forschungspreise des \\ Lungenfibrose e.V. für junge ILD- Forscher
}

Der Lungenfibrose e.V. schreibt anlässlich des 60. Kongresses der DGP im nächsten Jahr zwei Forschungspreise zu interstitiellen Lungenerkrankungen (ILD) im Allgemeinen, insbesondere zur Idiopathischen Lungenfibrose (IPF) aus.

Die Preise dienen der Förderung der Wissenschaft in diesem Gebiet.

Sie werden einmal für den besten grundlagenwissenschaftlichen und einmal für den besten klinischen Abstract über IPF bzw. ILD ausgeschrieben und sind mit jeweils 1500,00 Euro dotiert.

Abstracts zu anderen Themen wie COPD, TB, Asthma usw. werden nicht berücksichtigt.

Die aktuelle Arbeit darf zu keinem anderen Preis eingereicht sein.

Die Preise werden anlässlich des DGPKongresses 2019 in München vergeben.

Der/die Bewerber/in Erstautor/in darf muss höchstens 40 Jahre alt sein und wird gebeten, seine/ihre Abstracts bis zum 31. Januar 2019 im PDF-Format (max. 5MB) mit einer Publikationsliste und Lebenslauf an d.kauschka@lungenfibrose.de zu schicken.

Die Bewertung der Abstracts erfolgt durch drei unabhängige, namhafte Kollegen und berücksichtigt die Kriterien: 1. Originalität; 2. Methodologische Qualität; 3. Klinische bzw. wissenschaftliche Relevanz.

Dagmar Kauschka 1. Vorsitzende PD Dr. Francesco Bonella

2. stellvertr. Vorsitzender 